Delft University of Technology

\title{
Standards for UAS - Acceptable Means of Compliance for Low Risk SORA Operations
}

Cain, Sebastian; Torens, Christoph; Juchmann, Patrick; Volkert, Andreas; Tomasello, Filippo; Natale, Matteo; Vreeken, Joost; Ribeiro, M.J.; Ellerbroek, J.; More Authors

DOI

10.2514/6.2021-1177

Publication date

2021

Document Version

Final published version

Published in

AIAA Scitech 2021 Forum

\section{Citation (APA)}

Cain, S., Torens, C., Juchmann, P., Volkert, A., Tomasello, F., Natale, M., Vreeken, J., Ribeiro, M. J., Ellerbroek, J., \& More Authors (2021). Standards for UAS - Acceptable Means of Compliance for Low Risk SORA Operations. In AIAA Scitech 2021 Forum: 11-15 \& 19-21 January 2021Virtual/online event [AIAA 2021-1177] (AIAA Scitech 2021 Forum). American Institute of Aeronautics and Astronautics Inc. (AIAA). https://doi.org/10.2514/6.2021-1177

Important note

To cite this publication, please use the final published version (if applicable).

Please check the document version above.

\section{Copyright}

Other than for strictly personal use, it is not permitted to download, forward or distribute the text or part of it, without the consent of the author(s) and/or copyright holder(s), unless the work is under an open content license such as Creative Commons.

\section{Takedown policy}

Please contact us and provide details if you believe this document breaches copyrights.

We will remove access to the work immediately and investigate your claim. 
Green Open Access added to TU Delft Institutional Repository

'You share, we take care!' - Taverne project

https://www.openaccess.nl/en/you-share-we-take-care

Otherwise as indicated in the copyright section: the publisher is the copyright holder of this work and the author uses the Dutch legislation to make this work public. 


\title{
Standards for UAS - Acceptable Means of Compliance for Low Risk SORA Operations
}

\author{
Sebastian Cain ${ }^{1}$, Christoph Torens ${ }^{2}$, Patrick Juchmann ${ }^{3}$, Andreas Volkert ${ }^{4}$, \\ DLR - German Aerospace Center, Braunschweig, 38108, Germany \\ Filippo Tomasello ${ }^{5}$, Matteo Natale ${ }^{6}$, \\ EuroUSC, Italia Ltd, Roma, 00193, Italy \\ Joost Vreeken ${ }^{7}$, Tom van Birgelen ${ }^{8}$ \\ NLR - Royal Netherlands Aerospace Centre, Amsterdam, 1059CM, The Netherlands \\ Marta Ribeiro $^{9}$, Joost Ellerbroek ${ }^{10}$ \\ Delft University of Technology, 2629 HS Delft, The Netherlands \\ Damiano Taurino ${ }^{11}$, Marco Ducci ${ }^{12}$ \\ Deep Blue srl, Rome, 00198, Italy
}

\begin{abstract}
Unmanned aircraft systems (UAS) have been in civil use for several years. A new risk-based approach to approval was developed by the Joint Authorities for Rulemaking of Unmanned Systems (JARUS) which relies on the so-called Specific Operations Risk Assessment (SORA) for the specific category. Operational authorization is based on the assessment using the SORA process, which evaluates the safety of the operation and not solely the aircraft design. However, to comply with the resulting mitigations it is necessary to convince authorities using "Acceptable Means of Compliance" (AMC). The goal of the European research project "AW-Drones" is to identify and assess existing standards as a possible AMC for the existing and upcoming regulations. The research in "AW-Drones" is performed by an international consortium of industry and research agencies. Additional stakeholders support the project, including the European Union Aviation Safety Agency (EASA) and other groups of experts, committees, and Standard Development Organizations (SDOs). In this paper, the approach and methodology to identify possible AMC for the SORA is described, including the current state of work. The results of the data collection step and the assessment are outlined. The used criteria are shown and the impact on the SORA process is discussed. An outlook will detail on remaining tasks. The dissemination of the work in a public database is presented that offers the results on AMC assessment directly to a drone operator.
\end{abstract}

\footnotetext{
${ }^{1}$ Research Scientist, Institute of Flight Systems, Department of Unmanned Aircraft, AIAA Member.

${ }^{2}$ Research Scientist, Institute of Flight Systems, Department of Unmanned Aircraft, AIAA Senior Member.

${ }^{3}$ Research Scientist, Institute of Flight Systems, Department of Safety Critical Systems \& Systems Engineering.

${ }^{4}$ Research Scientist, Institute of Flight Guidance, Department of Pilot Assistance.

${ }^{5}$ Senior Partner.

${ }^{6}$ Safety and Compliance Engineer.

${ }^{7}$ R\&D Engineer, Vertical Flight \& Aeroacoustics department.

${ }^{8}$ R\&D Engineer, Air Transport Safety Institute.

${ }^{9} \mathrm{PhD}$ Candidate, Control \& Simulation Department, Faculty of Aerospace Engineering.

${ }^{10}$ Assistant Professor, Control \& Simulation Department, Faculty of Aerospace Engineering.

${ }^{11}$ Project Manager, Remotely Piloted Aircraft Systems.

12 Project Manager, Remotely Piloted Aircraft Systems.
} 


$\begin{array}{ll}\text { AMC } & \text { Acceptable Means of Compliance } \\ \text { AEH } & \text { Airborne Electronic Hardware } \\ \text { CS } & \text { Certification Specification } \\ \text { EASA } & \text { European Union Aviation Safety Agency } \\ \text { EC } & \text { European Commission } \\ \text { EU } & \text { Europäische Union } \\ \text { HMI } & \text { Human Machine Interface } \\ \text { JARUS } & \text { Joint Authorities for Rulemaking on Unmanned Systems } \\ \text { MCA } & \text { Multi-Criteria Analysis } \\ \text { OSO } & \text { Operational Safety Objective } \\ \text { SDO } & \text { Standard Development Organizations } \\ \text { SAIL } & \text { Specific Assurance and Integrity Level } \\ \text { SORA } & \text { Specific Operations Risk Assessment } \\ \text { SW } & \text { Software } \\ \text { UAS } & \text { Unmanned Aircraft System }\end{array}$

\section{Introduction}

Unmanned aircraft systems (UAS) have been in civil use for several years. Regulations have been developed alongside the technical development of UAS. With increasing commercial interest, regulations are necessary to enable and facilitate economic growth. Without them credible business plans would not be feasible. With regulations 2019/947 and 2019/945 of the European Commission (EC), and subsequent amendments in 2020, a risk-based approach to UAS operations is established. The approach was conceived by the "Joint Authorities for Rulemaking of Unmanned Systems" (JARUS) and implemented in recommending the "open", "specific," and "certified" categories. Whilst the open category is covered by product development specifications (like CE marks), the certified category will be covered by expanding the system of certification specifications equivalent to manned aviation. For large range of operations between them, forming the specific category, JARUS recommends using the so-called Specific Operations Risk Assessment (SORA) methodology. With this process, the certifying authority approval is based on assessment of the safety linked to all aspects of the operation and not solely on the safety of the aircraft itself (i.e. not only airworthiness). The safety of the aircraft airworthiness is targeted by „Operational Safety Objectives" (OSO). However, using the same methodology based on OSOs, mitigations based on operational limitations may also be applied to alleviate the airworthiness requirements, while still protecting the safety of third parties. Therefore, the result of the SORA process is a combination of requirements for the aircraft and for the specific operation (e.g. organization of the operator, qualification of the crews, operational limitations, etc.). A remaining important deficit is that still no means are in place to show the fulfillment of these requirements. The material presented here shall make a step towards solving this issue and help operators, authorities, and other stakeholders finding these means. The work shown in this paper is completely published via the project website www.aw-drones.eu. Additionally, an online repository is build up to give applicants direct access to the information about possible means to comply with the regulation.

To comply with the newly developed requirements, it is necessary to demonstrate the implementation of proper mitigations to aviation authorities. Using "Acceptable Means of Compliance" (AMC), similar to traditional aviation, greatly facilitates approval by the authority. These AMC provide applicants guidance based on industry best practices on how to comply, not only with the regulation requiring the risk assessment, but also with the mitigations stemming from the SORA process. These mitigations are necessary to obtain regulatory approval for operations in the "specific" category.

Usually, the use and selection of any AMC has to be discussed and agreed with the authority, which would require time and effort, both for the authority and the applicant. However, EASA is empowered by the EU legislator to publish lists of AMCs to facilitate this selection and use. In fact, EASA published several AMCs, some for airworthiness of manned aircraft, but also for other domains of manned aviation (e.g. AMC to PartCAT). Nevertheless, a comprehensive list of UAS related AMCs is still missing. 
An important input to the work presented here are collections of standards that are developed or in development and connected to the UAS topics. These collections come from Standard Development Organizations (SDOs) and provide a large database to start the search for possible AMCs. The three most extensive are the ANSI „Standardisation Roadmap for Unmanned Aircraft Systems“, the ASTM „UAS Roadmap," and the EUSCG "Rolling Development Plan“. However, they do not link the material to the actual requirements coming from the regulatory process and thus give no incidence on how to comply. This is the gap where the here presented work comes into play. This paper gives the background about the work to find AMC to the SORA. Not all results of the analysis can be shown as the discussion and presentation would be too extensive. The idea herein is to give an overview and the approach of the work to involve interested persons into the project work or gain interest in the actual results. These results are publicly available via the mentioned online repository. A drone operator can directly access all assessment information there and filter the data for the aspect or requirement he is looking for. The repository can be found via the website or by directly accessing standards.aw-drones.eu. The goal is to build up a living online database that offers help and with upcoming functions gather feedback of the drone community.

\section{The AW-Drones Project}

The goal of the European research project "AW-Drones" (AW derived from airworthiness) is to identify and assess existing consensus-based industry standards, applicable not only to airworthiness but to any aviation domain, and recommend them as possible AMC to comply with the existing and upcoming regulations. The research in "AW-Drones" is performed by an international consortium of industry, qualified entities, and research agencies. Additional stakeholders support the project, i.e. EASA and also other groups of experts, committees, and SDOs.

The focus of the project is a two-step approach that is targeted to identify applicable AMC for the SORA risk classes SAIL I - IV (i.e. low and medium risk in the specific category). It is planned to expand this approach later to additional topics, such as U-space, higher risk operations and, if so requested by EASA, also AMC for the certified category and for higher levels of autonomy of UAS. The first step is to develop an overview by establishing a database of UAS related standards, structured by topical fields. The second step is mapping the standards produced to the requirements of the SORA process. The last step is an analysis of the suitability of each standard as an AMC for each of the risk classes using a set of weighted criteria. The result, nicknamed the "EU metastandard", is not a new standard, but a guide facilitating the selection of the appropriate standard by any stakeholder. Furthermore, AW-Drones provides also a gap analysis of missing or inappropriate standards for the SORA risk classes, which need to be corrected to become AMC. If gaps are identified, the project will develop guidance on the content of these gaps for consideration by EASA, other authorities, and SDOs.

AW-Drones is a 36 months (2019-2021) project funded by the European Commission in the framework of Horizon 2020, the biggest EU Research and Innovation program. The project supports the on-going EU regulatory process for the definition of technical rules, standards, and procedures for civilian drones to enable safe, environmentally sound, and reliable operations in the EU. This objective is met through four main strands of activity:

- Collect information on on-going and planned work with regards to technical rules, procedures, and standards developed for mass-market drones worldwide.

- Carry out a critical assessment/benchmarking of all collected data to identify best practices, gaps, and bottlenecks;

- Propose and validate a well-reasoned set of technical standards for each category of drone operations;

- Engage with key stakeholders and end-users, i.e. representatives of the whole drone value chain.

EC and EASA, together with the project consortium, considered the current regulatory needs at EU level and decided to give priority to the following areas:

- Year 1: Analysis of standards required to support effectively the Specific Operations Risk Assessment (SORA) methodology. In particular, AW-Drones looks at the mitigation strategies proposed by SORA in its Annexes $B$ and $E$. The project identifies to what extent supporting 
standards to implement those mitigations are available or need to be developed. The identification of these standards initially focuses on technical ones, but those that are more related to operations and procedures are be considered as well.

- Year 2: Analysis of standard supporting the development of U-space in Europe. In particular, all standards required to support the technical implementation of U-space services in both U1, U2, and U3 phases will be addressed.

- Year 3: Towards its end, AW-Drones will focus on standards needed to support the operation of highly automated UAS and to ensure that UAS can be operated safely in a variety of applications. Standards and principles needed for UAS certification for autonomous operations will be investigated.

\section{Method for AMC Analysis}

The first method structures the collected technical rules, standards, and procedures of the database. To do so, a thematic structure was introduced that consists of a system of major domains and keywords within each domain. These domains and keywords are assigned to each standard in the database. The domains are major topics while the keywords provide a finer structure within these domains. With this structure, a user may focus on a certain aspect or topic without having a link to the regulatory side. Also, with this system, the standards can be structured that are currently out of scope of the regulations. The structure is described in Section IV in more detail.

Besides the thematic structuring a direct connection to the SORA is included to reflect the requirements coming from the regulations. The risks associated with drone operations in the specific category must be assessed in order to obtain an operational authorization. To understand the assessment process some fundamental knowledge about the SORA is needed. Without going into greater detail, a short summary of the SORA process is given here: The process starts by detailing the anticipated operation. Based on this description it guides the applicant in assessing the ground and air risk, identify tactical mitigations, and determine the risk for that operation on the basis of six Specific Assurance and Integrity Levels (SAIL). Depending on the SAIL, Operational Safety Objectives must be met and adjacent area and air space needs to be considered. At the different steps in this process, reference is made to standards accepted by the aviation authority, which have not been entirely specified yet. In order to systematically structure and subsequently assess standards that may support the SORA process, two sequential steps were applied.

The identified requirements from the SORA process are:

- 24 Operational Safety Objectives

- 3 Ground Risk Mitigations

- Strategic and Tactical Air Risk Mitigations, and

- Adjacent area/airspace considerations

All collected standards are mapped to the above items, in the process of the analysis this step is consequently referred to as mapping. This is done by evaluation of summaries and general data of the standard and drawing the connection in the database. The drawback of this approach is that standards addressing potential risks other than the ones considered by the SORA process may be missed. In other words, the standards are, with this method, purely evaluated regarding the SORA. The above described thematic approach can be used to cover the search for other aspects than the regulatory requirements. An important advantage is, that gaps of AMC (being SORA aspects for which no standards are identified) are easily identified. Furthermore, this proves to be an easy step to group standards for the assessment process regarding the requirements of the SORA.

The mapping is a first filter to identify possible AMC for the subsequent assessment methodology which bases on Multi-Criteria Analysis (MCA). This method evaluates the suitability of a standard as possible AMC by evaluating different criteria. Each criterion represents a certain aspect and all the ratings for the 
criteria are then combined to a resulting score. The exact approach and the criteria defined for the SORA process are described in Section $\mathrm{V}$ in more detail.

For a standard mapped to a certain requirement, the resulting weighted score indicates its usability. From this scoring, the following conclusions can be drawn:

- A standard corresponding to a requirement has a high score and is proposed as AMC. In case of partial coverage, the gaps must be indicated.

- A standard corresponding to a requirement that has a medium score is listed as possible AMC subject to decision by authority. In case of partial coverage, the gaps must be indicated.

- A standard corresponding to a requirement has a low score. Possible applicable standards from manned aviation and other industries are proposed, or a recommendation to amend the standard is provided. In case of partial coverage, the gaps must be indicated.

If a standard is mapped to a requirement but not found to be satisfyingly fulfilling the core criteria of this requirements the MCA is not performed as the standard is not a suitable AMC. The mapping indication from the first step is then removed to be in-line with the assessment results. Figure 1 summarizes the analysis process with the example requirements and criteria for the SORA. The blue elements are the input into the analysis. The analysis of a standards to be a possible AMC is shown in green, the analysis of gaps is identified in red. The steps of the process are explained more detailed in the next sections.

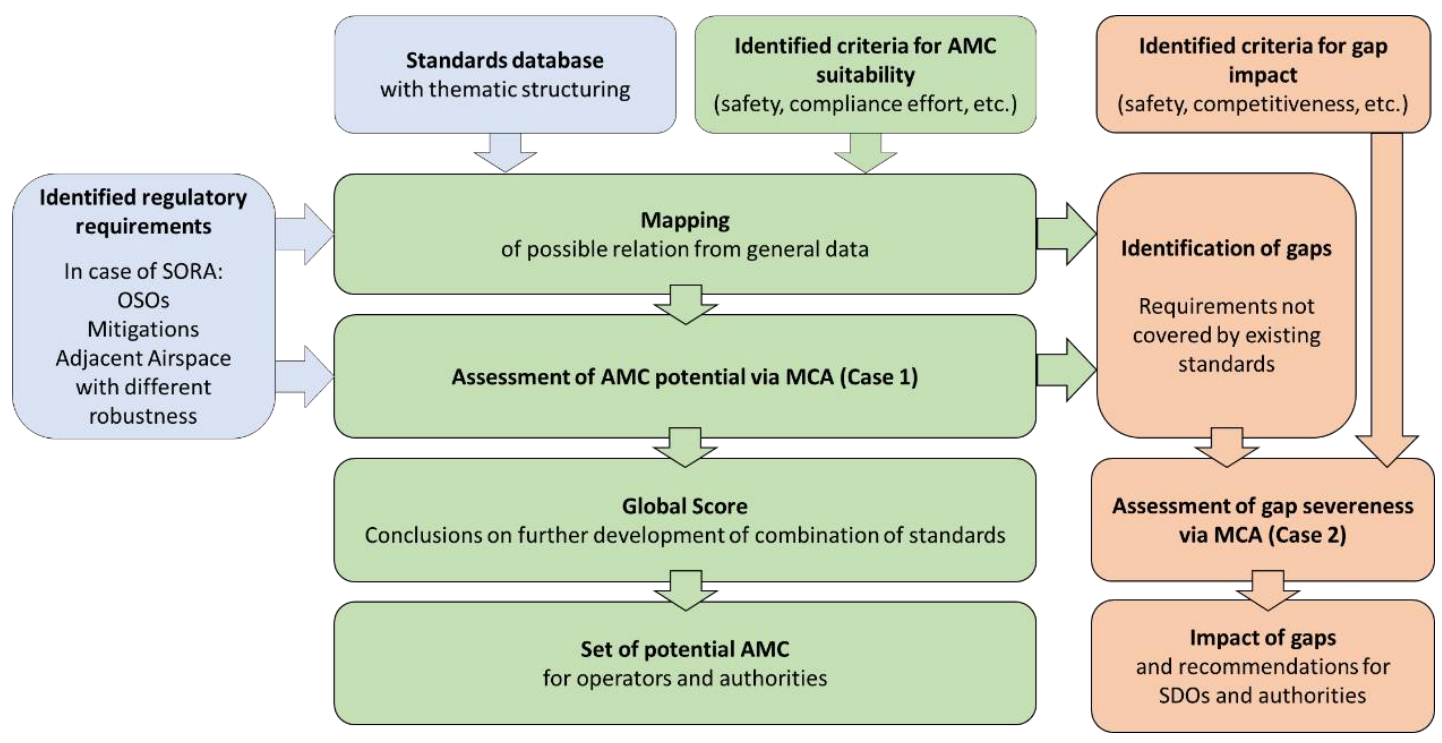

Figure 1: Structure of the information for the standards database

\section{Data Collection and Mapping}

The data collection and mapping step uses existing documents summarizing UAS related standards and combines these with expert knowledge of all project partners and stakeholders to set up a standards database. The available collections on subsets of standards are namely:

- EUSCG Rolling development plan

- ANSI Standardization Roadmap for Unmanned Aircraft Systems

- $\quad$ ASTM UAS Roadmap

- Smaller collections of other applicable standard organizations and bodies (EUROCAE, ISO, DIN, SAE, ...) 
All standards that are named in one of these collections, documents, and roadmaps have been researched and combined in a list of more than 650 documents. To be able to filter this list, additional information was added to the individual entries. The result is a comprehensive searchable database of UAS standards with additional meta-information, see Figure 1. The information within the database can be grouped into 3 sections.

The first section is standard specific information, general data (green), which includes information on the document type, the title of the document, the publishing organization, the publication status as well as a brief description. Also, the structuring with topics mentioned before consisting of domains and keywords are included here for each standard. The domains are general topics identified to be covering all UAS aspects and are suitable to structure all the material. They were discussed and iterated with experts from authorities and stakeholders. These domains are e.g. U-space, initial airworthiness, or operations. The subsequent keywords are more detailed aspects within the domains like detect and avoid, system safety assessment, or UAS operator training. The second section is the drone category (blue). Following EASA, all drone operation fall into one of the 3 categories: open, specific, and certified. The third meta-data section is information on the relation to the requirements identified from the SORA process (yellow). This section is related to the specific category and the performance of the SORA only. The information contained here is the aforementioned mapping. It is performed as a first rough step to find AMC. If a standard is supposed to affect a requirement in this section, it is marked for the next assessment step. As explained in section III the data contained in this section is iteratively updated to be in line with the assessment results. This means a standard is quickly mapped in the database to requirements it might be related to. If the assessment explained in the next section results in finding the standard not suitable for the requirement, the database is updated with this information.

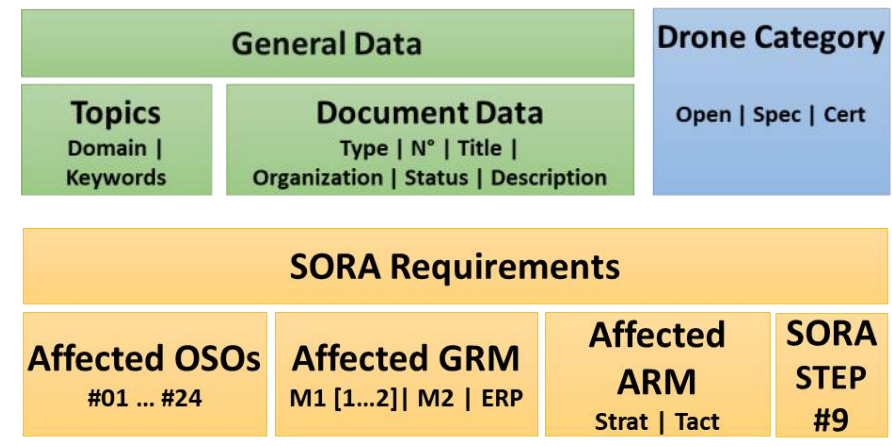

Figure 2: Structure of the information for the standards database

\section{Assessment of Standards and Gaps}

Both, the data collection and the assessment of the standards are carried out iteratively over the course of the three years of the project. It starts with the creation and further extension of a standards database, followed by the mapping of standards to requirements as first step. The next step is to investigate if an AMC is applicable to the specific category with the OSOs and mitigations required by SORA. In case no suitable standard is identified, the impact of this gap concerning for example the competitiveness of drone industry or safety is assessed as well.

In practice, three different cases of analysis are considered:

- CASE 1: one or more standards that are suitable to comply with a given requirement have been identified

- CASE 2: there is no standard fully covering a given requirement, thus a gap is identified

- CASE 3: a standard that does not map to any requirement has been identified ("orphan" standard).

For the first case, the assessed standards are ranked in terms of their suitability as AMC with drone regulations or SORA OSOs, yielding a final weighted score. The ranking is performed using an assessment methodology based on the principles of the MCA. Standards with high scores are proposed as AMC, while standards with medium scores are listed as possible AMC subject to decision by authority, in case of the 
SORA process of course the EASA. In the case of a partial coverage, gaps are identified and evaluated via a set of different criteria to rank them and quantify the consequences of the gap, in order to identify priorities and possible recommendations.

\section{Suitability analysis as AMC (Case 1)}

Beside assessing the applicability of a standard to a specific SORA requirement, the evaluation is extended beyond this aspect to cover a more general set of criteria.

The MCA is an analytical methodology employed to compare and rank standards (or anything else) when multiple factors must be considered. The assessment for the SORA evaluates each standard on the basis of the following criteria:

- Maturity of standard

- Type of standard

- Effectiveness to fulfil a SORA requirement

- Cost of compliance (to apply the standard to a specific level of robustness)

- Environmental impact

- Impact on EU industry competitiveness (e.g. does compliance to the standard potentially imply a disadvantage or advantage in international markets)

- Social acceptance (e.g. does compliance potentially generate an increase of social acceptance by containing aspects important for the public)

Eventually, a total weighted score of a standard is obtained via the MCA through the following method:

- A rating system is defined for each criterion to qualitatively express the magnitude of the effect of an option on the applicable aspect.

- To allow for the combination of criteria, which are expressed in qualitative terms and difficult to combine, non-dimensional numerical scores are defined for each rating system, in order to numerically express the results produced in the previous step (for example, very negative $=-2$, negative $=-1$, no effect $=0$, positive $=1$, very positive $=2$ ).

- Each criterion is given a weight factor proportional to the expected impact of the criteria (relative to the other criteria). For example, the "effectiveness to fulfil the SORA requirement," being of paramount importance, holds the highest impact on the evaluation.

- The final weighted score for each criterion is given by the product of their weight and the score; the weighted scores are summed up to produce a global score. This global score enables the immediate comparison of all identified standard related to each OSO.

The result of the described process shall enable conclusions on the applicability of the standard for this specific requirement. A standard with high final score will be considered a good AMC. If no standard provides high score, the lower rated standards may be considered and conclusions for improvement are outlined. The conclusion may be further development of the standard or a combination with other material. The scale for deriving this recommendation is shown in Figure 3

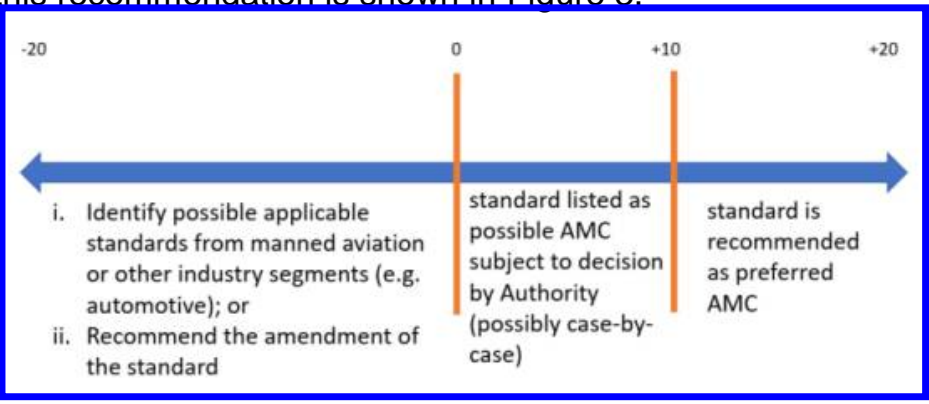

Figure 3: Final score and derived conclusions for each assessed standard

\section{Effectiveness to fulfil SORA requirements}


The SORA constitutes that different requirements (e.g. OSOs) are fulfilled with different levels of robustness (low, medium, high) for different risk categories (e.g. SAIL). Also, each OSO compliance is separated into integrity and assurance aspect. Each standard identified as potential AMC to the SORA is subsequently assessed for the different levels of robustness and separately for integrity and assurance to evaluate the extent to which the requirements are covered.

The scope of the analysis is limited, in the first iteration of the project to the level of robustness required up to SAIL IV operations (i.e. low and medium risks in the specific category). The assessment outlines the effectiveness of each standard to fulfil the SORA requirement for each level of robustness, yielding a list of gaps where there are no standards fully covering the whole requirement. Gaps may in fact arise even if a standard has a full coverage, while not covering the full range of UAS designs (such as standards only for fixed wing UAS).

\section{Gap assessment (Case 2)}

In the case that a SORA requirement is only partially covered by a standard, any gap to complete coverage is identified and evaluated. This evaluation is again performed by applying different criteria to provide a ranking system in order to help prioritise the necessary actions and derive possible recommendations for SDOs and/or authorities. The total weighted score of a gap is the again obtained via the MCA analogous to the suitability analysis described before. The criteria are expressed with qualitatively ratings which are merged with numerical scores and respective weights to calculate the final score.

The criteria identified for the gap assessment analyses are:

- Effect on safety

- Cost of compliance to the requirements with a lack of standard coverage

- Environmental impact or possible damage

- Impact on EU industry competitiveness

- Effect on social acceptance by possibly increased risks

The final score on the gap is evaluated with the scale shown in Fiqure 4.

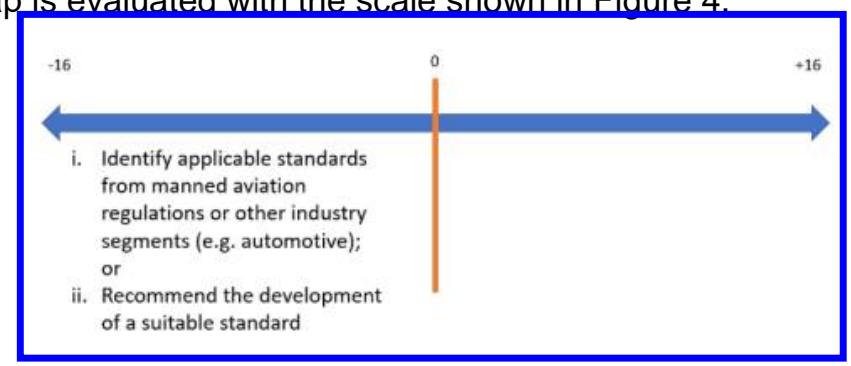

Figure 4: Resulting rating of an identified gap to comply with a requirement

A negative number indicates that the gap is severe. Conversely a positive number indicates that the need to develop additional guidance/standard is not considered an immediate priority.

Section VI below provides the conclusions and highlights the main recommendations that were derived from the outlined analysis during the first year of the project.

\section{Conclusion on Standards and Identified Gaps}

The following gaps and conclusions are based on the existing published standards available during the first half of the project. The standards related to UAS are continuously developed or may be replaced or expanded with new, more applicable standards. Thus, the results reflect the state at the time the analysis was carried out. The work is an ongoing, iterative process and new developments are continuously monitored by the project team. More recently results are continuously published by the project and made available in the reports on the project website and the online repository.

For all SORA requirements, applicable up to SAIL IV, there is at least a partial coverage from existing standards. However, only a small number achieve full coverage. All SORA requirements and coverage indications are presented in Tables 1-5. Each row represents a certain requirement and is divided into the 
different levels of robustness (Low, Medium, and High) in the columns. The coloring represents the coverage of each requirement. A gray shading indicates that a standard is not required for that level of robustness for this requirement. A yellow shading indicates partial robustness coverage. A green shading indicates full robustness coverage. When higher robustness levels are not necessary for UAS operations up to SAIL IV, these are not referenced (this is the case for the High-level robustness for several of the SORA requirements).

Table 1: Strategic Mitigations for Ground Risk - Coverage Overview

\begin{tabular}{|l|c|c|c|}
\hline \multicolumn{1}{|c|}{ Requirement } & \multicolumn{3}{c|}{ Robustness Coverage } \\
\hline M1 - non tethered & L & M & H \\
\hline M1 - tethered & L & M & H \\
\hline M2 & L & M & H \\
\hline M3 & L & M & H \\
\hline
\end{tabular}

Table 2: Tactical Mitigation Performance Requirements (VLOS) - Coverage Overview

\begin{tabular}{|c|c|}
\hline Requirement & Robustness Coverage \\
\hline $\begin{array}{ll}\text { Tactical } & \text { Mitigations } \\
\text { VLOS } & \end{array}$ & \\
\hline
\end{tabular}

Table 3: Tactical Mitigations Performance Requirements (BVLOS) - Coverage Overview

\begin{tabular}{|l|l|l|l|l|}
\hline \multirow{2}{*}{ Function } & \multicolumn{4}{c|}{ Robustness Coverage } \\
\cline { 2 - 5 } & Arc-a & Arc-b & Arc-c & Arc-d \\
\hline Detect & & & & \\
\hline Decide & & & & \\
\hline Command & & & & \\
\hline Execute & & & & \\
\hline Feedback Loop & & & & \\
\hline Integrity & & & & \\
\hline
\end{tabular}

Table 4: OSOs - Coverage Overview

\begin{tabular}{|l|c|c|c|}
\hline \multicolumn{1}{|c|}{ Requirement } & \multicolumn{3}{c|}{$\begin{array}{c}\text { Robustness } \\
\text { Coverage }\end{array}$} \\
\hline OSO \#01 & $\mathrm{L}$ & $\mathrm{M}$ & $\mathrm{H}$ \\
\hline OSO \#02 & $\mathrm{L}$ & $\mathrm{M}$ & - \\
\hline OSO \#03 & $\mathrm{L}$ & $\mathrm{M}$ & - \\
\hline OSO \#04 & $\mathrm{L}$ & - & - \\
\hline OSO \#05 & $\mathrm{L}$ & $\mathrm{M}$ & - \\
\hline OSO \#06 & $\mathrm{L}$ & $\mathrm{M}$ & - \\
\hline OSO \#07 & $\mathrm{L}$ & $\mathrm{M}$ & - \\
\hline OSO \#08, \#11, \#14, \#21 & $\mathrm{L}$ & $\mathrm{M}$ & $\mathrm{H}$ \\
\hline OSO \#09, \#15, \#22 & $\mathrm{L}$ & $\mathrm{M}$ & - \\
\hline OSO \#10, \#12 & $\mathrm{L}$ & $\mathrm{M}$ & - \\
\hline OSO \#13 & $\mathrm{L}$ & $\mathrm{M}$ & $\mathrm{H}$ \\
\hline OSO \#16 & $\mathrm{L}$ & $\mathrm{M}$ & - \\
\hline OSO \#17 & $\mathrm{L}$ & $\mathrm{M}$ & - \\
\hline OSO \#18 & $\mathrm{L}$ & $\mathrm{M}$ & - \\
\hline OSO \#19 & $\mathrm{L}$ & $\mathrm{M}$ & - \\
\hline OSO \#20 & $\mathrm{L}$ & $\mathrm{M}$ & - \\
\hline OSO \#23 & $\mathrm{L}$ & $\mathrm{M}$ & - \\
\hline OSO \#24 & $\mathrm{L}$ & $\mathrm{M}$ & $\mathrm{H}$ \\
\hline
\end{tabular}


Table 5: Adjacent Airspace - Coverage Overview

\begin{tabular}{|c|c|c|}
\hline Requirement & \multicolumn{2}{|c|}{ Robustness Coverage } \\
\hline Adjacent Airspace & 1 & 2 \\
\hline
\end{tabular}

Tables 6 to 9 display an overview of the means of compliance that are missing to fully support each SORA requirement. After each table, the impact of such gaps and consequent recommendations to SDOs and authorities are discussed.

The gaps have been classified into four different categories, to better highlight their nature:

- Guidelines: guidance material supporting the UAS operator in how to show compliance to the SORA requirement

- Procedures: specific instructions and protocols associated with UAS operations

- Technical: related to the design of the UAS, its components and/or external services

- Training: how to conduct the trainings and structure training material for personnel involved in UAS operations

Regarding the severity of the gaps based on the scoring system described in Section $\mathrm{V}$, the following shading is used:

- An orange shading indicates a severe gap (with a score smaller or equal to -5).

- A red shading indicates a very severe gap (with a score score smaller or equal to -10).

- A white shading indicates that a gap is not considered severe.

Naturally, very severe and severe gaps should be tackled first by SDOs or authorities as these represent higher safety risks and compliance costs.

Table 6: Strategic Mitigations for Ground Risk - Gap Overview

\begin{tabular}{|c|c|c|}
\hline Req & Gap & Area \\
\hline \multirow{5}{*}{ M1 } & \multirow{3}{*}{ G } & Common approach for the definition of the ground risk buffer \\
\hline & & Evaluation of people at risk \\
\hline & & Requirements for real time population data \\
\hline & $P$ & Installation/maintenance of tether \\
\hline & $\mathrm{Te}$ & Mechanical characteristics of the line \\
\hline \multirow{5}{*}{ M2 } & \multirow{2}{*}{ G } & Standard automation technique system activation \\
\hline & & Reduction of effects of impact dynamics and post impact hazards \\
\hline & \multirow{2}{*}{$P$} & Contingency/emergency procedures \\
\hline & & Installation/maintenance procedures \\
\hline & $\operatorname{Tr}$ & Ground impact measures \\
\hline \multirow{3}{*}{ M3 } & \multirow{2}{*}{ G } & Emergency Response Plan suitable for UAS operations \\
\hline & & Reducing number of people at risk \\
\hline & $\operatorname{Tr}$ & Coping with UAS emergencies \\
\hline
\end{tabular}

The most serious gaps on strategic mitigations for ground risk (Table 6) are as follows:

- The absence of specific requirements on how to correctly evaluate the risk to third parties and impact hazard on ground with and without a mitigation to reduce such risk may result in important factors being overlooked.

- The absence of specific guidelines for the definition of the ground risk buffer may have negative impact on safety as adequate margins might not be retained in all flight conditions.

- The lack of standards for tether makes it hard to evaluate the adequacy of the tether for the UAS.

In regard to the previous gaps, the following is advised for further development of AMC: 
- Guidelines defining how to evaluate the number of people at risk for operators should be developed ad hoc for operational, technical and administrative topics.

- Contingency and emergency conditions need to be standardised in order to define procedures handle technical issues.

- Guidelines for checklists will reduce the time necessary for authorities to evaluate operator's documentation.

- A training program dedicated to the skills needed to handle emergency situations safely should be developed.

Table 7: Tactical Mitigations Performance Requirements - Gap Overview

\begin{tabular}{|c|c|l|}
\hline Req & Gap & \multicolumn{1}{c|}{ Area } \\
\hline \multirow{2}{*}{ VLOS } & \multirow{2}{*}{ G } & Guidance to produce a documented deconfliction scheme \\
\cline { 3 - 3 } & & Procedures/protocols to support the deconfliction scheme \\
\hline \multirow{2}{*}{ BVLOS } & \multirow{2}{*}{ Te } & Detection and Avoid (DAA) specifically for small UAS \\
\cline { 3 - 3 } & & Small UAS above very low level (VLL) \\
\hline
\end{tabular}

The gaps on tactical mitigations, both for VLOS and BVLOS, (Table 7) have the following impact:

- The lack of a standardized way to develop a VLOS deconfliction scheme might compromise the air risks for UAS operations

- Reliable and standardized DAA procedures are needed to avoid collisions between unmanned and manned traffic. Although small drones have a limited size and mass, several studies indicate that these collisions may still result in serious damages to manned traffic [1]-[3].

- The absence of recognised DAA standards makes it impossible to carry out operations associated with Air Risk Class Arc-d and BVLOS [4]. This leads to the necessity to segregate airspace (which strongly restricts the operational volume).

It is therefore recommended to:

- Develop dedicated guidance material to help operators produce a VLOS/E-VLOS deconfliction scheme. These should adequately define methods and criteria used to avoid incoming traffic, along with the procedures that are in place to support such scheme.

- Development of standardized requirements and tests for detect and avoid technology allowing for the safe integration of UAS operations into the civil airspace.

Table 8: OSOs - Gap Overview

\begin{tabular}{|c|c|c|}
\hline Req & Gap & Area \\
\hline $\begin{array}{c}\text { OSO } \\
\# 1\end{array}$ & G & Minimum requirements in terms of structure, post-holders, or categories of operations \\
\hline $\begin{array}{c}\text { OSO } \\
\# 4\end{array}$ & G & $\begin{array}{l}\text { Current standards are too demanding and not applicable to low and medium } \\
\text { robustness and SAIL IV operations }\end{array}$ \\
\hline $\begin{array}{l}\text { OSO } \\
\# 6\end{array}$ & Te & Communication functionalities where needed/relevant \\
\hline \multirow{6}{*}{$\begin{array}{l}\text { OSO } \\
\# 08, \\
\# 11, \\
\# 14, \\
\# 21\end{array}$} & G & Crew interactions with other entities involved in UAS operations \\
\hline & \multirow{4}{*}{$P$} & $\begin{array}{l}\text { Operational procedures for UAS and the associated elements. Some UAS } \\
\text { configurations may require specific standards. }\end{array}$ \\
\hline & & Contingency/emergency procedures \\
\hline & & Operational checklists for staff \\
\hline & & Procedures to manage human errors \\
\hline & $\mathrm{Tr}$ & Training the Remote Crew with Crew Resource Management knowledge \\
\hline \multirow{2}{*}{$\begin{array}{l}\text { OSO } \\
\# 09\end{array}$} & \multirow[t]{2}{*}{$\operatorname{Tr}$} & $\begin{array}{l}\text { Personnel, other than remote pilot, in charge of duties essential to the management of } \\
\text { the flight }\end{array}$ \\
\hline & & Non-regulated professions (e.g. supporting personnel, payload operator, flight \\
\hline
\end{tabular}




\begin{tabular}{|c|c|c|}
\hline $\begin{array}{l}\# 15, \\
\# 22\end{array}$ & & dispatcher) \\
\hline \multirow{3}{*}{$\begin{array}{l}\text { OSO } \\
\# 13\end{array}$} & G & Defining GNSS performance adequacy for drone operations \\
\hline & \multirow{2}{*}{$\mathrm{P}$} & Standardized procedures for the monitoring of external services \\
\hline & & Testing verifying GNSS performance \\
\hline \multirow{2}{*}{$\begin{array}{l}\text { OSO } \\
\# 16\end{array}$} & $\mathrm{G}$ & Assignment of crew tasks and establishing step-by-step communications \\
\hline & $\mathrm{Te}$ & Communication devices suitable for drone crews \\
\hline \multirow{2}{*}{$\begin{array}{l}\text { OSO } \\
\# 17\end{array}$} & \multirow{2}{*}{ G } & Criteria addressing fit conditions before or during duty times \\
\hline & & Fatigue Risk Management System \\
\hline $\begin{array}{l}\text { OSO } \\
\# 18\end{array}$ & $\mathrm{Te}$ & $\begin{array}{l}\text { Automatic protection of the flight envelope following remote pilot errors specifically for } \\
\text { small UAS }\end{array}$ \\
\hline \multirow{3}{*}{$\begin{array}{l}\text { OSO } \\
\# 19\end{array}$} & G & $\begin{array}{l}\text { Procedures and checklists for error recovery including a clear allocation of tasks } \\
\text { among the remote crew }\end{array}$ \\
\hline & Te & UAS design of solutions to recovery from human error \\
\hline & $\operatorname{Tr}$ & Visual observers, for safety reasons \\
\hline \multirow{2}{*}{$\begin{array}{l}\text { OSO } \\
\# 20\end{array}$} & G & $\begin{array}{l}\text { Human factors evaluation of UAS determining if the Human Machine Interface (HMI) } \\
\text { is appropriate for the operation }\end{array}$ \\
\hline & $\mathrm{Te}$ & Platform-independent HMI capabilities \\
\hline \multirow{2}{*}{$\begin{array}{l}\text { OSO } \\
\# 23\end{array}$} & \multirow{2}{*}{ G } & How to determine adequate environmental conditions for safe operations \\
\hline & & Current procedures are quite generic and do not provide sufficient guidance \\
\hline
\end{tabular}

The gaps on compliance with the OSOs (Table 8) have the following effects:

- The lack of standard procedures has a negative effect on cost development of UAS as well as operations, as each operator/manufacturer must research its own form of compliance.

- The lack of harmonized training requirements increases effort from operators and authorities in order to check personnel competences.

- The likelihood and effects of human error in the absence of standard procedures and adequate training is increased.

- The lack of standards for safety limits and contingency procedures may weaken the openness of the market for drone operations as a lack of safety will heavily decrease social acceptance.

- Adequate HMI is crucial to support UAS operations safety [5]. Without standards for design and development, it is hard to understand to what extent the available HMI is able to safely support UAS operations in terms of information presentation and human error.

- The lack of well-defined safe operating conditions for UAS could have adverse effects to the environment. In extreme cases, an accident could cause environmental pollution. This is not requirement of the SORA itself, however it might affect social acceptance.

It is recommended for the adequate SDOs and authorities to develop standards which directly cover the gaps identified. It should be considered that:

- Common approaches concerning the structure of standards should be developed. These enhance the safety of UAS operations, preventing misunderstandings and the overlooking of important factors.

- Human factors and training have been identified as key enablers for the drone market [6]-[10], and should thus be prioritized. Moreover, the training of remote crews should consider the inclusion of physical and mental conditions, as these can greatly affect the outcome of UAS operations.

- Currently, a considerable number of standards have a limited scope (e.g. restriction to MTOM less than $25 \mathrm{~kg}$ or only rotorcraft). A larger scope for newly created standards would be beneficial in order to reduce the number of standards each operator must consider.

Standards developed for the manned aviation can be too demanding for the UAS sector and are hardly applicable in practice, especially for small UAS. Similarity with manned aviation procedures can be 
beneficial in order to guarantee that all safety aspects are considered; however, effort of procedures should be properly scaled when directed at lower-risk UAS operations.

Regarding adjacent airspace (Table 9), there is a need for more material on how to demonstrate that the procedures, as well as errors from the SW and/or $\mathrm{AEH}$, do not result in an infringement of the adjacent areas on the ground and/or adjacent airspace.

Table 9: Adjacent Airspace - Gap Overview

\begin{tabular}{|c|c|c|}
\hline Req & Gap & Area \\
\hline \multirow{2}{*}{$\begin{array}{c}\text { Adjacent } \\
\text { Airspace }\end{array}$} & \multirow{2}{*}{$\mathrm{G}$} & $\begin{array}{c}\text { Preventing actions outside the operational volume after failure of the UAS or of } \\
\text { any external system }\end{array}$ \\
\cline { 3 - 3 } & & $\begin{array}{c}\text { Software (SW), Airborne Electronic Hardware (AEH) development assurance for } \\
\text { small UAS }\end{array}$ \\
\hline
\end{tabular}

\section{Expert Groups \& Dissemination}

During all the development phases of AW-Drones, experts, and stakeholders are involved and regularly informed, in order to verify the adherence of the project outcomes to the expectations of the drone community.

In particular, the project consortium fosters a tight cooperation with EASA and the Standard Development Organizations that is carried out through regular update meetings and collaborative workshops.

The involvement of a wider audience of drone stakeholders (manufacturers, operators, researchers, etc.) is achieved through the constitution of an external experts Advisory Board, that meets biannually and the organization of large yearly workshops open to the public.

The AW-Drones open repository is an online platform where users are able to easily identify relevant information from the AW-Drones database of standards and regulations. It addresses UAS operators directly and provides support whenever an AMC for a certain regulatory requirement is needed.

The online platform provides a single point of access to relevant information about:

- rules, procedures and technical standards developed for civilian drones;

- $\quad$ best practices, gaps, and bottlenecks;

- technical standard for each category of drone operations.

In particular, the repository contains the full and continuously updated information produced by the project activities described in Section IV, V, and VI. The information architecture and the whole web repository design is inspired by the principles of User Centered Design, with the aim to offer a good experience to the users and enable an easy access of drone operators to find AMC.

Apart from an open platform that can be used as information exchange (access, mine, exploit, reproduce, disseminate data), the AW-Drones repository also includes collaboration features (commenting, rating, adding and editing content, reviewing, etc.) that will enhance its use and purpose and will further support its sustainability even after the end of the AW-Drones project.

The AW-Drones open repository was recently released to the public and will be continuously expanded regarding its functionality. It is linked by the project website: https://www.aw-drones.eu/.

\section{Summary and Outlook}

This paper gives an overview on the work carried out in the AW-Drones project. A method is presented to analyse existing standards that are linked to the UAS domain. This first analysis was done with respect to identified requirements coming from the SORA process. Conclusions about their applicability for these requirements were drawn. The current status of this work, starting with the composition of a standards database, and then performing the analysis in two steps is shown. The first step is the so-called mapping, followed by the more detailed assessment. From this work, first deductions about possible AMC were found; some standards with high potential were identified and gaps for further development by SDOs were concluded. 
The work is published in an online repository to the public to help drone operators. This repository shall give guidance to find the best possible AMC to each regulatory requirement. Additionally, the data derived is presented to EASA in reports and sets of recommended standards in order to help finding acceptable standards and include the feedback from experts into the results. The authority may use this work as a basis to publish s standard set of AMCs in the next months.

The repository as well as mapping and assessment must be continuously updated to keep track with new development and will also be expanded during the project run. It is planned to apply the developed method to upcoming U-space regulations and furthermore to UAS related Certification Specifications (CS) that are currently under development by EASA for the specific category. Even for the certified category, an additional analysis of AMC is under discussion with EASA to achieve a status comparable to manned aviation. In this context, the work presented here, contributes to UAS certification.

\section{References}

[1] G. Olivares et al., "FAA sUAS COE Task A3 UAS Airborne Collision Hazard Severity Evaluation", Alliance for System Safety of UAS through Research Excellence (ASSURE), 2017. https://www.assureuas.org/projects/deliverables/a3/A3\%20Airbone\%20Collision.pdf

[2] R. J. Wallace et al., "Evaluating Small UAS Near Midair Collision Risk Using AeroScope and ADSB”, Int. J. Aviat. Aeronaut. Aerosp. Aeronaut. Aerosp., vol. 5, no. 4 Article 2, 2018. https://commons.erau.edu/cgi/viewcontent.cgi?article=1268\&context=ijaaa

[3] D. Jones, "Small Remotely Piloted Aircraft Systems (drones): Mid-Air Collision Study", Department for Transport, Military Aviation Authority, British Airline Pilots Association, 2017.

https://assets.publishing.service.gov.uk/government/uploads/system/uploads/attachment_data/file/918811/sm all-remotely-piloted-aircraft-systems-drones-mid-air-collision-study.pdf

[4] M. Askelson, H. Cathey, "Small UAS Detect and Avoid Requirements Necessary for Limited Beyond Visual Line of Sight (BVLOS) Operations", University of North Dakota and New Mexico State University, 2017.

https://www.assureuas.org/projects/deliverables/a2/Final_Report_A2_sUAS_BVLOS_Requirements.pdf

[5] D. A. Vincenzi, B. A. Terwilliger, D. C. Ison, "Unmanned Aerial System (UAS) Human-Machine Interfaces: New Paradigms in Command and Control," Procedia Manuf., vol. 3, pp. 920-927, 2015. https://www.assureuas.org/projects/deliverables/a2/Final_Report_A2_sUAS_BVLOS_Requirements.pdf

[6] SESAR Joint Undertaking, "European drones outlook study : Unlocking the value for Europe", 2017. https://op.europa.eu/en/publication-detail/-/publication/93d90664-28b3-11e7-ab65-01aa75ed71a1/languageen/format-PDF/source-38537167

-[7] K. W. Williams, "8. Human Factors Implications of Unmanned Aircraft Accidents: Flight-Control Problems", Human Factors of Remotely Operated Vehicles (Advances in Human Performance and Cognitive Engineering Research), Vol. 7, pp. 105-116, 2006.

https://doi.org/10.1016/S1479-3601(05)07008-6

[8] J. S. Mccarley, C. D. Wickens, "Human Factors Implications of UAVs in the National Airspace", Institute of Aviation, Aviation Human Factors Division, University of Illinois at Urbana-Champaign, 2006.

http://www.tc.faa.gov/logistics/grants/pdf/2004/04-G-032.pdf

[9] A.-D. Schmitz, R. Pelchen-Medwed, "PODIUM Demonstration report for VLD - Part IV - Human Performance Assessment Report", 2019.

https://www.sesarju.eu/sites/default/files/documents/projects/783230_D1_2_PODIUM_Human_Performance_ Assessment_Report\%20(1_0).pdf

[10] A. Hobbs, B. Lyall, "Human Factors Guidelines for Unmanned Aircraft System Ground Control Stations. Preliminary Guidelines 1.0 Contractor Report prepared for NASA UAS in the NAS Project", 2015.

https://hsi.arc.nasa.gov/publications/GCS_HF\%20_Prelim_Guidelines_Hobbs_Lyall.pdf 